\title{
Fuzzy Clustering Based Approach for Prediction of Level of Severity of Faults in Software Systems
}

\author{
Kritika Gupta and Sandeep Kang
}

\begin{abstract}
There is lot of work done in prediction of the fault proneness of the software systems. But, it is the severity of the faults that is more important than number of faults existing in the developed system as the major faults matters most for a developer and those major faults needs immediate attention. As, Neural networks, which have been already applied in software engineering applications to build reliability growth models predict the gross change or reusability metrics. In which majority of faults are found in a few of its modules so there is a need to investigate the modules that are affected severely as compared to other modules and proper maintenance need to be done in time especially for the critical applications. In this present work, hybrid fuzzy-Genetic Algorithm and fuzzy clustering based technique to be studied. Fuzzy clustering Based techniques are also discussed for the comparative analysis in order to predict level of impact of faults in NASA's public domain defect dataset. Predicting faults in the software life cycle can be used to improve software process control and achieve high software reliability. The results show that when the best prediction techniques are evaluated.
\end{abstract}

Index Terms-Neural network, software faults, software metric, fuzzy clustering, decision tree, k-means, software quality.

\section{INTRODUCTION}

Faults in software systems continue to be a major problem. A software bug is an error, flaw, mistake, failure, or fault in a computer program that prevents it from behaving as intended (e.g., producing an incorrect result). A software fault is a defect that causes software failure in an executable product. In software engineering, the non-conformance of software to its requirements is commonly called a bug. Most bugs arise from mistakes and errors made by people in either a program's source code or its design, and a few are caused by compilers producing incorrect code. Knowing the causes of possible defects as well as identifying general software process areas that may need attention from the initialization of a project could save money, time and work. The possibility of early estimating the potential faultiness of software could help on planning, controlling and executing software development activities. Prediction of fault prone modules in software development process and mostly used the metric based approach with machine learning

Manuscript received July 9, 2011; revised August 12, 2011.

The authors are in CGC, Landran, Punjab; (e- mail: kriti.kanu07@gmail.com). techniques to model the fault prediction in the software modules.

Software Metrics

Metrics is defined as "The continuous application of measurement based techniques to the software development process and its products to supply meaningful and timely management information, together with the use of those techniques to improve that process and its products". Software metrics is all about measurement and these are applicable to all the phases of software development life cycle from initiation to maintenance.

The IEEE Standard Glossary defines metric as a "Quantitative measure of degree to which a system, component or process possess a given attribute. Amount of incoming pulses needed to activate a neuron also change. This behavior allows the $\mathrm{NN}$ to learn. We can train a neural network to perform a particular function by adjusting the values of the connections (weights) between elements. Commonly neural networks are adjusted, or trained, so that a particular input leads to a specific target output.

The main aim of this work is to model the impact of faults in function based software modules. The main objectives are described as follows:

- To find the structural code and design attributes of software systems

- Find the best algorithms that can be used to model impact of faults in object oriented i.e. the predict the level of impact of the faults in the software system

This paper is organized as follows: Section two describes the Methodology part of work done, which shows the steps used in order to reach the objectives and carry out the results. In the section three, results of the implementation are discussed. In the last section, on the basis of the discussion various Conclusions are drawn and the future scope for the present work is discussed.

\section{II.PROPOSED METHODOLOGY}

The methodology consists of the following steps:

\section{A. Find the Structural Code and Design Attributes}

The first step is to find the structural code and design attributes of software systems i.e. software metrics. The real-time defect data sets are taken from the NASA's MDP (Metric Data Program) data repository http://mdp.ivv.nasa.gov/. The dataset is related to the safety critical software systems 
being developed by NASA.

\section{B. Collection \& Processing of Metric Values}

The suitable metrics like product module metrics out of these data sets are considered. The term product is used referring to module level data. The term metrics data applies to any finite numeric values, which describe measured qualities and characteristics of a product. The term product refers to anything to which defect data and metrics data can be associated. In most cases products will be synonymous with code related items such a functions and systems/sub-systems. The description of the module level metrics are shown in Table 1.

\section{C.Analyze and refine metrics the metric values}

In the next step table of module levels metrics PC4_product_module_metrics is joined with PC4_defect_product_relations and thereafter again the join operation of the resultant table is performed with PC4_static_defect_data. An Entity-Relationship diagram relates Modules to Defects and Defects to Severity of Defects is shown in figure 1.

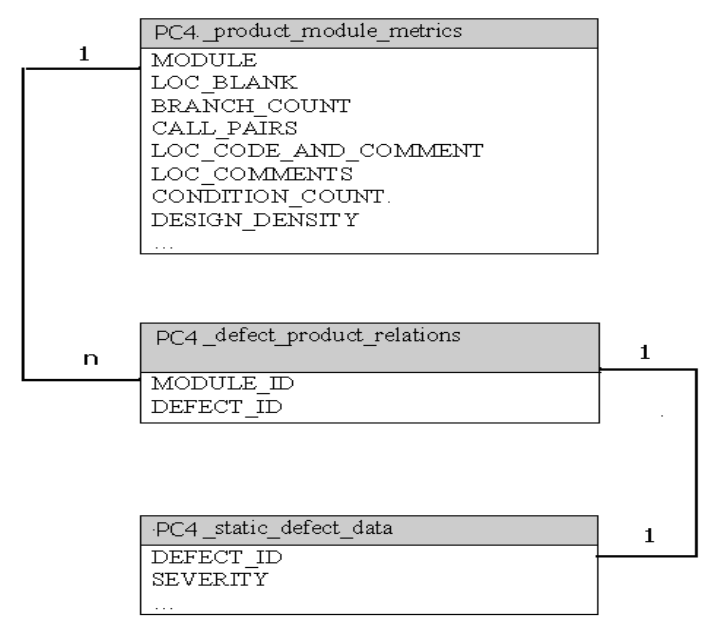

Fig. 1 An entity-relationship diagram relates modules to defects and defects to severity of defects

In the figure 1 the MODULE_ID is the unique numeric identifier of the module and DEFECT_ID is the unique numeric identifier of the associated defect. The SEVERITY field in the PC4_static_defect_data table shows the value that quantifies the impact of the defect on the overall environment in the range of 1 to 5 . Where, 1 means most severe and 5 being least severe. For example, severity 1 may imply that the defect caused a loss of functionality without a workaround where severity 5 may mean that the impact is superficial and did not cause any major disruptions to the system.

\section{D.Explore Fuzzy Clustering Technique}

It is very important to find the suitable algorithm for modeling of software components into different levels of fault severity in software systems. The following fuzzy clustering algorithm is experimented

TABLE I :MODULE LEVEL METRICS

\begin{tabular}{|c|c|}
\hline Metric Name & Description \\
\hline MODULE & Unique numeric identifier of the product. \\
\hline LOC_BLANK & Number of blank lines in a module. \\
\hline BRANCH_COUNT & Branch count metrics. \\
\hline CALL_PAIRS & $\begin{array}{l}\text { Number of calls to other functions in a } \\
\text { module. }\end{array}$ \\
\hline $\begin{array}{l}\text { LOC_CODE_AND_COMMEN } \\
\mathrm{T}\end{array}$ & $\begin{array}{l}\text { Number of lines which contain both code } \\
\& \text { comment in a module. }\end{array}$ \\
\hline LOC_COMMENTS & $\begin{array}{l}\text { Number of lines of comments in a } \\
\text { module. }\end{array}$ \\
\hline CONDITION_COUNT & $\begin{array}{l}\text { Number of conditionals in a given } \\
\text { module. }\end{array}$ \\
\hline $\begin{array}{l}\text { CYCLOMATIC_COMPLEXIT } \\
\text { Y }\end{array}$ & Cyclomatic complexity of a module. \\
\hline CYCLOMATIC_DENSITY & $\begin{array}{l}\text { Ratio of the module's cyclomatic } \\
\text { complexity to its length in NCSLOC. }\end{array}$ \\
\hline DECISION_COUNT & $\begin{array}{l}\text { Number of decision points in a given } \\
\text { module. }\end{array}$ \\
\hline DECISION_DENSITY & Calculated as: Cond / Decision. \\
\hline DESIGN_COMPLEXITY & Design complexity of a module. \\
\hline DESIGN_DENSITY & $\begin{array}{l}\text { Design density is calculated as: } \\
\operatorname{iv}(G) / v(G) \text {. }\end{array}$ \\
\hline EDGE_COUNT & $\begin{array}{l}\text { Number of edges found in a given } \\
\text { module. }\end{array}$ \\
\hline ERROR_COUNT & $\begin{array}{l}\text { Number of defects associated with a } \\
\text { module. }\end{array}$ \\
\hline ERROR_DENSITY & $\begin{array}{l}\text { Number of defects per } 1000 \text { lines of code } \\
\text { for a module }\end{array}$ \\
\hline ESSENTIAL_COMPLEXITY & Essential complexity of a module. \\
\hline ESSENTIAL_DENSITY & $\begin{array}{l}\text { Essential density is calculated as: } \\
(\operatorname{ev}(G)-1) /(v(G)-1) \text {. }\end{array}$ \\
\hline LOC_EXECUTABLE & $\begin{array}{l}\text { Number of lines of executable code for a } \\
\text { module }\end{array}$ \\
\hline PARAMETER_COUNT & Number of parameters to a given module. \\
\hline $\begin{array}{l}\text { GLOBAL_DATA_COMPLEXI } \\
\text { TY }\end{array}$ & $\begin{array}{l}\text { Global Data Complexity quantifies the } \\
\text { cyclomatic complexity of a module's } \\
\text { structure as it relates to global/parameter } \\
\text { data. }\end{array}$ \\
\hline GLOBAL_DATA_DENSITY & $\begin{array}{l}\text { Global Data density is calculated as: } \\
\operatorname{gdv}(\mathrm{G}) / \mathrm{v}(\mathrm{G}) \text {. }\end{array}$ \\
\hline HALSTEAD_CONTENT & Halstead length content of a module. \\
\hline HALSTEAD_DIFFICULTY & Halstead difficulty metric of a module. \\
\hline HALSTEAD_EFFORT & Halstead effort metric of a module. \\
\hline HALSTEAD_ERROR_EST & Halstead error estimate metric. \\
\hline HALSTEAD_LENGTH & Halstead length metric of a module. \\
\hline HALSTEAD_LEVEL & Halstead level metric of a module. \\
\hline HALSTEAD_PROG_TIME & $\begin{array}{l}\text { Halstead programming time metric of a } \\
\text { module. }\end{array}$ \\
\hline HALSTEAD_VOLUME & Halstead volume metric of a module. \\
\hline $\begin{array}{l}\text { MULTIPLE_CONDITION_CO } \\
\text { UNT }\end{array}$ & $\begin{array}{l}\text { Number of multiple conditions that exist } \\
\text { within a module. }\end{array}$ \\
\hline NODE_COUNT & $\begin{array}{l}\text { Number of nodes found in a given } \\
\text { module. }\end{array}$ \\
\hline NUM_OPERANDS & $\begin{array}{l}\text { The number of operands contained in a } \\
\text { module. }\end{array}$ \\
\hline NUM_OPERATORS & $\begin{array}{l}\text { The number of operators contained in a } \\
\text { module. }\end{array}$ \\
\hline NUM_UNIQUE_OPERANDS & $\begin{array}{l}\text { The number of unique operands contained } \\
\text { in a module. }\end{array}$ \\
\hline NUM_UNIQUE_OPERATORS & $\begin{array}{l}\text { The number of unique operators } \\
\text { contained in a module. }\end{array}$ \\
\hline NUMBER_OF_LINES & Number of lines in a module. \\
\hline $\begin{array}{l}\text { PATHOLOGICAL_COMPLEX } \\
\text { ITY }\end{array}$ & $\begin{array}{l}\text { A measure of the degree to which a } \\
\text { module contains extremely unstructured } \\
\text { constructs. }\end{array}$ \\
\hline PERCENT_COMMENTS & Percentage of the code that is comments. \\
\hline LOC_TOTAL & $\begin{array}{l}\text { The total number of lines for a given } \\
\text { module. }\end{array}$ \\
\hline
\end{tabular}


Fuzzy clustering:-

Clustering can be a very effective technique to identify natural groupings in data from a large data set, thereby allowing concise representation of relationships embedded in the data. In our study, clustering allows us to group software modules into faulty and non-faulty categories hence allowing for easier understandability. The purpose of clustering is to identify natural groupings of data from a large data set to produce a concise representation of a system's behavior. Fuzzy Logic Toolbox command line function fem starts with an initial guess for the cluster centers, which are intended to mark the mean location of each cluster. Main Steps of fuzzy clustering algorithm:-

Step 1: Calculate the input data to be clusters.

$$
X_{i j} i=1,2, \ldots ., n ; j=1,2, \ldots ., m
$$

$n$ is the number of data

$m$ is the type of data

Step 2: Set the variables value:

$$
\begin{aligned}
& i-\quad r_{j,} j=1,2, \ldots . ., m \\
& i i-\quad \eta q u a s h \text { factor } \\
& i i i-\quad \varepsilon^{*} \text { acceptratio } \\
& i v-\quad \text { Erejectratio } \\
& v-X_{j, \min } \\
& v i-\quad X_{j \max }
\end{aligned}
$$

Step 3: Set the normal data value based on $X_{j-\min }$ dan $X_{j-\max }$ use with the following model:

Step 4: Set the potential of each data point by the formula:

$$
\begin{array}{r}
X_{i j}^{n o r m}=\frac{X_{i j}-X_{j-\min }}{X_{j-\min }-X_{j-\min }}, i=1,2, \ldots ., n ; j=1,2, \ldots ., m \\
a=1, \text { revise } a=n
\end{array}
$$

Step 5: Set the highest potential value of data:

$$
\begin{aligned}
& M={ }_{\max }\left[p_{i}^{\prime} \mid i=1,2, \ldots . n\right] \\
& h=i, \text { so that } D_{i}=M
\end{aligned}
$$

Step 6: Set cluster centre and update the potential value that correspond to another data:

$$
\begin{aligned}
& i-C n t=[] \\
& i i-V_{j}=X_{h j} ; j=1,2, \ldots ., m \\
& i i i-C=0 \text { (number of clusters) } \\
& i v-C n d=1 \\
& v-z=m \\
& v i-D o C n d \neq 0 \text { and } Z \neq 0
\end{aligned}
$$

Step 7: Put the real data:

$$
C n t_{i j}=C n t_{i j} *\left(X_{j-\max }-X_{j-\min }\right)+X_{j-\min }
$$

Step 8: Set the cluster sigma:

$$
\sigma_{j}=\frac{r_{j}\left(X_{j-\max }-X_{j-\min }\right.}{\sqrt{8}}
$$

\section{Comparison of Algorithms}

The comparisons are made on the basis of the more accuracy and least value of MAE and RMSE error values. Accuracy value of the prediction model is the major criteria used for comparison. The mean absolute error is chosen as the standard error. The technique having lower value of mean absolute error is chosen as the best fault prediction technique.

- Mean absolute error

Mean absolute error, MAE is the average of the difference between predicted and actual value in all test cases; it is the average prediction error [13]. The formula for calculating MAE is given in equation 7 .

$$
\frac{\left|a_{1}-c_{1}\right|+\left|a_{2}-c_{2}\right|+\ldots+\left|a_{n}-c_{n}\right|}{n}
$$

Assuming that the actual output is a, expected output is c.

- Root mean-squared error

RMSE is frequently used measure of differences between values predicted by a model or estimator and the values actually observed from the thing being modeled or estimated [13]. It is just the square root of the mean square error as shown in equation 8 .

$$
\sqrt{\frac{\left(a_{1}-c_{1}\right)^{2}+\left(a_{2}-c_{2}\right)^{2}+\ldots+\left(a_{n}-c_{n}\right)^{2}}{n}}
$$

The mean-squared error is one of the most commonly used measures of success for numeric prediction. This value is computed by taking the average of the squared differences between each computed value and its corresponding correct value. The root mean-squared error is simply the square root of the mean-squared-error. The root mean-squared error gives the error value the same dimensionality as the actual and predicted values.

Fuzzy logic is an effective paradigm to handle imprecision. It can be used to take fuzzy or imprecise observations for inputs and yet arrive at crisp and precise values for outputs. Also, the Fuzzy Inference System (FIS) is a simple and commonsensical way to build systems without using complex analytical equations.

Here, fuzzy logic will be employed to capture the broad categories identified during clustering into a Fuzzy Inference System (FIS). The FIS will then act as a model that will reflect the relationship between the different input parameters.

\section{RESUltS AND DiscusSION}

The real-time defect data set used is taken from the NASA's MDP (Metric Data Program) data repository, the details of that dataset contains 178 modules of C Programming language with different values of software fault severity labeled present as 1, 2 and 3. The severity level 4 and 5 are not present in the PC4 dataset. So, the level 1 represents the highest severity, level 2 represents the medium and level 3 represents the minor fault that can be overlooked to save time. Details of the type of 
faults existing in different number of modules of the Dataset are shown in bar chart of figure land numeric values are tabulated in table 2 .

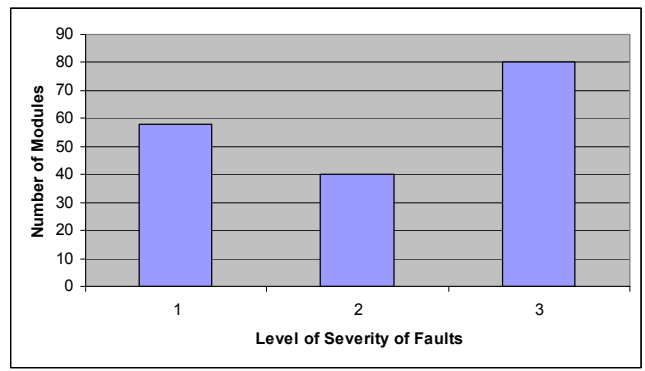

Fig. 2. Graphical representation of different Severity of Faults in Modules TABLE II: DETAILS OF THE DIFFERENT SEVERITY OF FAULTS IN MODULES

\begin{tabular}{|c|c|}
\hline Severity Level & Count of Modules \\
\hline 1 & 58 \\
\hline 2 & 40 \\
\hline 3 & 80 \\
\hline
\end{tabular}

The algorithms are evaluated on the basis of the following criteria:

The developed software computes the mean absolute error, root mean squared error, relative absolute error and root relative squared error. However, the most commonly reported error is the mean absolute error and root mean squared error. The root mean squared error is more sensitive to outliers in the data than the mean absolute error. In order to minimize the effect of outliers, mean absolute error is chosen as the standard error. The prediction technique having least value of mean absolute error is chosen as the best prediction technique.

Mean absolute error, MAE is the average of the difference between predicted and actual value in all test cases. The root mean-squared error i.e. RMSE is simply the square root of the mean-squared-error. The root mean-squared error gives the error value as the same dimensionality as the actual and predicted values.

The mean-squared error is one of the most commonly used measures of success for numeric prediction. This value is computed by taking the average of the squared differences between each computed value and its corresponding correct value. The MAE and the RMSE can be used together to diagnose the variation in the errors in a set of forecasts. The RMSE will always be larger or equal to the MAE.

The greater difference between them, the greater the variance in the individual errors in the sample. If root mean squared error is equal to mean absolute error, then all the errors are of the same magnitude. Both root mean squared error and mean absolute error can range from 0 to $\infty$.

MAE and RMSE are negatively-oriented scores and lower values are better. So, algorithm with least value of mean absolute error is considered as the best algorithm. The training phase performance of the Batch Gadient without momentum, Batch Gadient with momentum, Variable Learning Rate without momentum, Variable Learning Rate with momentum and Resilient Backpropagation is shown in figure 3-7 respectively.

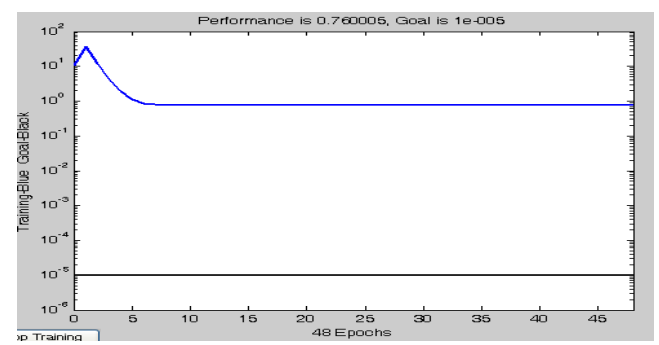

Fig. 3. Training Performance of Batch Gradient Descent

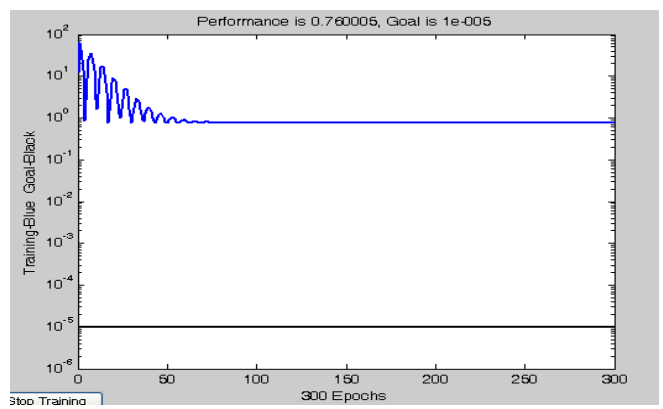

Fig. 4. Training performance of batch gradient descent with momentum

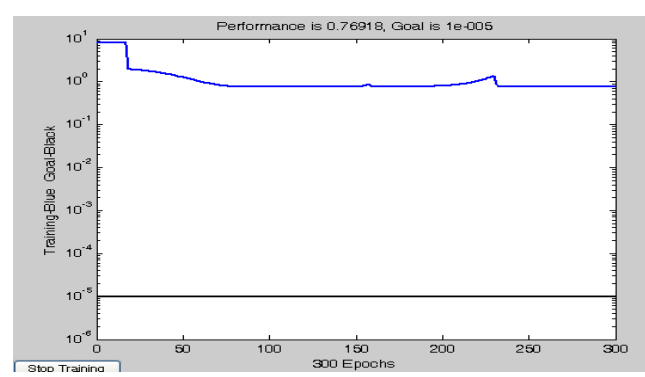

Fig. 5. Training performance of variable learning rate without momentum algorithm

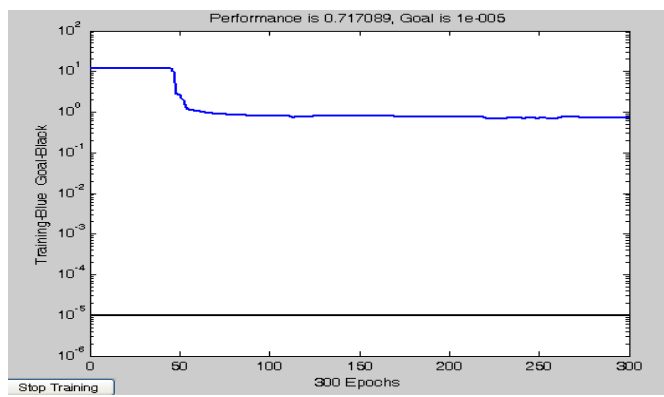

Fig. 6. Training performance of variable learning rate with momentum algorithm

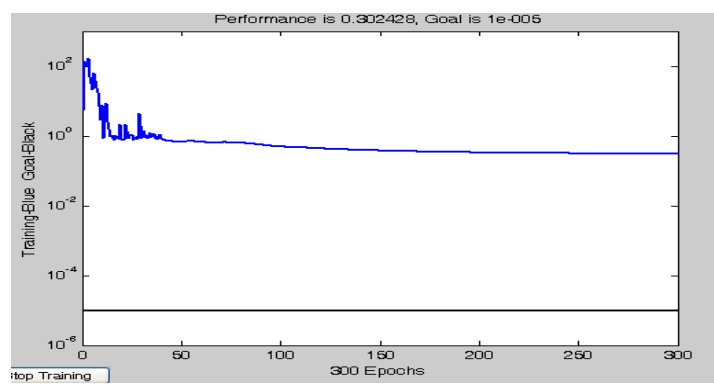

Fig. 7. Training performance of resilient back propagation 
In the present work the five Neural Network based algorithms experimented in Matlab 7.4 and after the training each trained network is tested with testing dataset of 15 values derived from the PC4 dataset. The overall testing performance of the different algorithms is shown in table 3.The results reveal that the fuzzy clustering algorithm have outperformed all other algorithm under study with $0.2982,0.4200$ and $100 \%$ as MAE, RMSE and Accuracy values respectively.

TABLE III: PERFORMANCE RESULTS OF DIFFERENT RESILIENT BACK PROPAGATION AND FUZZY CLUSTERING ALGORITHMS

\begin{tabular}{|c|l|c|c|c|}
\hline $\begin{array}{c}\text { Sr. } \\
\text { No. }\end{array}$ & Algorithm & $\boldsymbol{M A E}$ & $\boldsymbol{R M S E}$ & $\begin{array}{c}\text { Accuracy } \\
\%\end{array}$ \\
\hline 1 & Fuzzy clustering & 0.2982 & 0.4200 & 100 \\
\hline
\end{tabular}

\section{CONCLUSION}

According to [17], on comparing the classes of WEKA's machine learning algorithms, it is observed that fuzzy clustering algorithm is best prediction techniques as compared with other classes of machine learning algorithms in modeling of severity of faults in software systems. In [19] resilient backpropagation based technique was also introduced. The purposed fuzzy clustering based technique on the basis of testing data with 100, 0.2982 and 0.4200 as Accuracy, Mean Absolute Error and Root Mean Square Error values.

It is therefore, concluded the model is implemented and the best algorithm for modeling of the function based software modules into different level of severity of impact of the fault is found to be fuzzy clustering technique. It is the best prediction technique as compare to the other machine learning technique. it gives more accurate result as compare to the other machine learning techniques. Hence, the proposed algorithm can be used to identify modules that have major faults and require immediate attention.

\section{REFERENCES}

[1] A. Abrand and H. Nguyenkim, "Analysis of Maintenance Work Categories through Measurement," Proceedings of IEEE Conference on Software Maintenance, Sorrento, Italy: IEEE, (1991), 104-113.

[2] H. Kung and C. Hsu, "Software Maintenance Life Cycle Model, "Proceedings International Conference on Software Maintenance, IEEE (1998), Washington D.C.
[3] H. Kung, "Quantitative Method to Determine Software Maintenance Life Cycle," Proceedings of the 20th IEEE International Conference on Software Maintenance, IEEE, (2004).

[4] T. M. Pigoski, "Practical software maintenance," Wiley computer publishing, (1997).

[5] V. Gibson and J. Senn, "System Structure and Software Maintenance Performance," Comm. of ACM, vol. 27, no. 3, Mar. (1989), pp. 347-358.

[6] M. Deodhar (2002), "Prediction Model and the Size Factor for Fault-proneness of Object Oriented Systems," MS Thesis, Michigan Tech. University, Dec. 2002.

[7] P. Bellini, (2005), "Comparing Fault-Proneness Estimation Models," 10th IEEE International Conference on Engineering of Complex Computer Systems (ICECCS'05), vol. 0, 2005, pp. 205-214.

[8] T. M. Khoshgoftaar, K. Gao, and R. M. Szabo ( 2001), “An Application of Zero-Inflated Poisson Regression for Software Fault Prediction. Software Reliability Engineering," ISSRE 2001. Proceedings of 12th International Symposium on, 27-30 Nov. (2001), pp: 66 -73.

[9] U. B. Venkata, Challagulla, B. Farokh Bastani, and I-Ling Yen, "Empirical Assessment of machine Learning based Software Defect Prediction Techniques," Proceedings of the 10th IEEE International Workshop on Object-Oriented Real-Time Dependable Systems (WORDS'05), IEEE (2005)

[10] T. M. Khoshgoftaar and J. C. Munson, (1990). "Predicting Software Development Errors using Complexity Metrics," IEEE Journal on Selected Areas in Communications, 8(2): 253 -261.

[11] T. Menzies, A. K. Ammar, Nikora, and S. Stefano, (2003), "How Simple is Software Defect Prediction," Journal of Empirical Software Engineering, October (2003).

[12] K. Eman, S. Benlarbi, N. Goel and S. Rai, (2001), "Comparing case-based reasoning classifiers for predicting high risk software components," Journal of Systems Software, 55(3): $301-310$.

[13] J. P. Hudepohl, S. J. Aud, T. M. Khoshgoftaar, E. B. Allen, and J. E. Mayrand, (1996), "Software Metrics and Models on the Desktop", IEEE Software, 13(5): 56-60.

[14] T. M. Khoshgoftaar, E. B. Allen, K. S. Kalaichelvan, and N. Goel, (1996), "Early quality prediction: a case study in telecommunications," IEEE Software (1996), 13(1): 65-71.

[15] T. M. Khoshgoftaar, and N. Seliya, (2002), "Tree-based software quality estimation models for fault prediction," METRICS 2002, the Eighth IIIE Symposium on Software Metrics, pp: 203-214.

[16] N. Seliya, T. M. Khoshgoftaar, S. Zhong, (2005), "Analyzing software quality with limited fault-proneness defect data," Ninth IEEE international Symposium, Oct 12-14, (2005).

[17] J. C. Munson and T. M. Khoshgoftaar, (1992), "The detection of fault-prone programs," IEEE Transactions on Software Engineering, 18(5): 423-433.

[18] S. P. Singh, S. Kumar, and H. Singh, (2007), "Intelligence System for Software Maintenance Severity Prediction," Journal of Computer Science, Vol. 3 (5), pp. 281-288, 2007.

[19] Reidmiller, Proceedings of the IEEE Int. Conf. on NN (ICNN) San Francisco, 1993, pp. 586-591

[20] S. Parvinder, U. S. Malhotra, and E. Ardil, "Predicting the Impact of the Defect on the Overall Environment in Function Based Systems," World Academy Of Science, Engineering And Technology, issue 56, Aug 2009, pp. $140-43$

[21] N. Seliya, T. M. Khoshgoftaar, and S. Zhong, "Analyzing software quality with limited fault-proneness defect data," Ninth IEEE international Symposium on 12-14 Oct, (2005).

[22] J. h Zhou, S. Parvinder Sandhu, and S. Rani "A Neural Network Based Approach for Modeling of Severity of Defects in Function Based Software Systems" (2010) IEEE. 